\title{
Isognomon bicolor (C.B. Adams) (Bivalvia, Isognomonidae): primeiro registro para o Brasil, redescrição da espécie e considerações sobre a ocorrência e distribuição de Isognomon na costa brasileira
}

\author{
Osmar Domaneschi ${ }^{1}$ \\ Claudio Mantovani Martins ${ }^{2}$
}

\begin{abstract}
Isognomon bicolor (C.B. Adams) (Bivalvia, Isognomonidae): first record to the Brazilian littoral, redescription of the species and comments on the occurrence and distribution of Isognomon in the Brazilian coast. The bivalve family Isognomonidae is represented in the Western Atlantic by the living genera Crenatula Lamarck, 1804 and Isognomon Solander, 1786. I. alatus (Gmelin, 1791) and $I$. radiatus (Anton, 1839) are the only Isognomonidae referred to the Brazilian malacofauna. The present work refers to the first record and geographic distribution of $I$. bicolor along the Brazilian littoral, and presents a re-description of the species based on shell characters, which include those of the prodissoconch. The occurrence of $I$. alatus and $I$. radiatus along the Brazilian littoral could not be confirmed, despite the intensive search for these Isognomonidae from Rio Grande do Norte through Rio Grande do Sul.
\end{abstract}

KEY WORDS. Bivalvia, Isognomonidae, Isognomon bicolor, re-description, geographic distribution, Brazil

A família Isognomonidae Woodring, 1925 reúne cerca de 20 espécies em dois únicos gêneros viventes, a maioria de distribuição tropical, epifaunal bissada (Isognomon Solander, 1786), ou em associação com esponjas (Crenatula Lamarck, 1804) (Boss 1982; COAN et al. 2000). Isognomon está representado no Atlântico Oeste por I. alatus (Gmelin, 1791), I. radiatus (Anton, 1839) e I. bicolor (C.B. Adams, 1845) (WARMKE \& ABBOTT 1962; АвBOTT 1974; HuMFREY 1975; EMMERSON \& JACOBSON 1976), das quais I. bicolor não é mencionada para o Brasil.

MATTHEWS \& KEMPF (1970) são os primeiros a registrar um Isognomonidae para o Brasil com o encontro de "Isognomon cf. alatus" (sic) no Atol das Rocas, "não assinalada no Arquipélago de Fernando de Noronha". Após esse registro, Aввотt (1974), ABBotT \& DANCE (1983), SABELI (1985), MERLANO \& HEGEDUS (1994), ABBOTT \& MORRIS (1995) mencionam essa espécie para o Brasil, sem precisar localidades, enquanto RIOS $(1970,1975,1985)$ e COSEL (1986) referem-se a I. alatus como restrita ao Atol das Rocas no Brasil. TARASCONI (1989) identifica

1) Departamento de Zoologia, Instituto de Biociências, Universidade de São Paulo. Caixa Postal 11461, 05422-970 São Paulo, São Paulo, Brasil. E-mail: domanesc @usp.br. Bolsista CNPq.

2) Curso de Pós-Graduação, Departamento de Zoologia, Instituto de Biociências, Universidade de São Paulo. Financiamento FAPESP. 
como "I. alatus Gmelin, 1791" (sic) o Isognomonidae que coletou em Santa Catarina, região sul do Brasil, e RIOS (1994) estende a distribuição para a região sudeste ao mencionar a ocorrência dessa espécie de São Sebastião, em São Paulo, até Santa Catarina. Isognomon radiatus é outro Isognomonidae citado para o Brasil por AвBOTT (1974), АвBOTT \& DANCE (1983) e ABBOTT \& MORRIS (1995), igualmente sem indicação de localidades e da fonte do registro.

Concomitante ao registro de I. alatus por RIOS (1994), para a região sudeste, os autores do presente estudo eram notificados da presença recente na Praia de Barequeçaba, São Sebastião, de populações muito densas de um bivalve ocupando zonas dos costões tradicionalmente habitadas pelos bivalves Brachidontes Swainson, 1840 e ostreídeos. A análise de espécimes dessas novas populações revelou tratar-se de Isognomon, gênero não registrado para a região de São Sebastião desde o levantamento malacológico pioneiro por IHERING (1897), até o mais recente por MigotTo et al. (1993). Caracteres externos da concha permitiram descartar, de imediato, tratar-se de $I$. radiatus, enquanto semelhanças com $I$. alatus, recém registrada para essa região por RIOS (1994), restringiam-se apenas aos aspectos externos.

A tentativa de identificação através do caráter expresso pelo número absoluto mínimo e máximo de sulcos ligamentais da charneira, como referido na literatura, não se afigurou de valor taxonômico dada as grandes discrepâncias nos números registrados para uma mesma espécie (ex.: I. alatus) por TRUEMAN (1954), ABBOTT (1954, 1974), WARMKE \& ABBOTT (1962), HuMFREY (1975), EMERSON \& JACOBSON (1976), SIUNG (1980) e RIOS (1994), e pelo fato de ABBOTT (1974) e EMERSON \& JACOBSON (1976) registrarem números mínimo e máximo idênticos para espécies distintas (ex.: I. bicolor e $I$. radiatus). TRUEMAN (1954) é o único autor a se referir ao número de sulcos ligamentais, relacionando-o ao comprimento e crescimento da charneira em I. alatus, sem contudo tecer consideração sobre a importância desse registro.

Essa análise comparativa suscitou a hipótese de que as populações recém instaladas em São Sebastião poderiam ser de I. bicolor, espécie muito similar a $I$. alatus segundo ABBOTT (1974) e HUMFREY (1975), e que a espécie se constitui numa nova ocorrência para a localidade, e para o Brasil.

Variações intraespecífica e deformações na concha em decorrência do hábito de vida em grandes adensamentos ou da ocupação de espaços restritos em fendas ou cavidades dificultam a distinção entre I. alatus e I. bicolor. Esse quadro é agravado por insuficiências nas descrições originais, na definição e/ou ilustração de caracteres diagnósticos, apontando a necessidade de redescrição desse material.

A verificação da hipótese de se tratar de $I$. bicolor, a elaboração de redescrição detalhada da espécie caso se confirme a suspeita, bem como a realização de pesquisas de campo ao longo do litoral brasileiro para se determinar sua atual distribuição geográfica são objetivos do presente trabalho. A investigação da presença de $I$. alatus e de $I$. radiatus nos locais visitados constitui objetivo complementar, buscando confirmar a ocorrência e/ou ampliar a distribuição geográfica destas duas últimas espécies. 


\section{MATERIAL E MÉTODOS}

No período de 1994 a 1999 os autores realizaram buscas e coletas de representantes de Isognomonidae no Arquipélago de Fernando de Noronha, no litoral de Estados brasileiros das regiões nordeste (Rio Grande do Norte, Pernambuco, Bahia), sudeste (Rio de Janeiro, São Paulo) e sul (Santa Catarina, Rio Grande do Sul). Não foi visitado o Atol das Rocas, localidade de proveniência dos espécimes que permitiram a MATTHEWS \& KEMPF (1970) fazerem o primeiro registro do gênero Isognomon para o Brasil.

Para o estudo comparativo com os espécimes de Isognomon sp. coletados pelos autores, espécimes de I. bicolor e I. alatus foram obtidos por empréstimos e/ou doações feitos pelos conquiliologistas Luiz Trinchão (LT) e José Carlos Tarasconi (JCT), por pesquisadores, e pelos museus: Museu de Zoologia, Universidade de São Paulo (MZUSP), Museu Nacional, Universidade Federal do Rio de Janeiro (MNRJ), Museu Oceanográfico "Eliezer de Carvalho Rios", Fundação Universidade de Rio Grande (MORG), "The Bailey-Matthews Shell Museum", Flórida, E.U.A. (BMSM), "Zoologisk Museum", Universidade de Copenhague, Dinamarca (ZMUC) e "Museum of Comparative Zoology", Harvard, E.U.A. (MCZ).

O material original de "Isognomon cf. alatus" (sic), coligido por MATTHEWS \& KeMPF (1970) no Atol das Rocas, não pôde ser examinado por se encontrar extraviado da coleção do Laboratório de Ciências do Mar da Universidade Federal do Ceará (LABOMAR-UFCE) (Helena Matthews-Cascon, UFCE, com. pess.). O material testemunho que serviu para o registro de $I$. alatus na região sudeste brasileira por RiOs (1994), não foi localizado nos acervos dos museus contatados, bem como esse autor não informa a instituição depositária.

Exemplares do gênero Isognomon provenientes de todas localidades brasileiras onde se constatou sua ocorrência, quer coletados pelos autores, quer por colaboradores, foram identificados até o nível de espécie e depositados nos acervos do Museu de Zoologia da Universidade de São Paulo (MZUSP) e do "American Museum of Natural History" (AMNH), E.U.A. como referência de ocorrência da espécie para o Brasil. Os lotes provenientes das coletas efetuadas pelos autores e por colaboradores estão identificados com $(*)$ ou $(+)$, respectivamente, entre os "materiais examinados", apresentados na seqüência.

Avalia-se pela primeira vez a importância da relação entre o "número de sulcos ligamentais versus extensão da charneira" para a distinção entre I. alatus e Isognomon sp. aqui registrada. Os resultados dessa análise são apresentados na tabela I, onde a extensão e o número de sulcos ligamentais da charneira de 87 espécimes de Isognomon sp. (47 coletados pelos autores, mais 40 entre os provenientes de museus e os recebidos por doação), perfazendo uma série completa de crescimento, e 50 espécimes de I. alatus (42 recebidos por empréstimo e mais oito relativos aos examinados por TRUEMAN (1954)), estão agrupados dentro das respectivas espécies por classes de extensão de charneira. Os dados de TRUEMAN (1954) relativos a oito espécimes variando de 6,4 a 14,3 $\mathrm{mm}$ de comprimento de charneira foram incorporados à tabela I para se aumentar a amplitude de variação dessa dimensão na amostra, bem como o número de indivíduos analisados, uma vez 
que cinco desses espécimes têm charneira mais curta que os 42 recebidos por empréstimo para o presente estudo. A despeito dessa incorporação, não se conseguiu reunir uma série de crescimento completa de $I$. alatus.

A altura da concha, determinada pela distância entre duas paralelas, uma tangencial à linha da charneira, a outra tangencial à margem ventral da concha (Fig. 1), é o parâmetro adotado para expressar o "tamanho" dos espécimes analisados, como é freqüente na literatura pertinente a Isognomonidae.

Para a análise comparativa do material de Isognomon coletado pelos autores e mais aquele obtido por empréstimo, com o lectótipo de I. bicolor, o síntipo de I. alatus, e respectivas descrições originais, foram consideradas exclusivamente as características conquiliológicas como forma, ornamentação, altura, elementos da face interna das valvas e número de sulcos ligamentais em relação à extensão da charneira.

\section{RESULTADOS E DISCUSSÃO}

\section{Isognomon bicolor (C.B. Adams, 1845)}

Figs 1-9

Perna bicolor C.B. Adams, 1845: 9 [lectótipo, Jamaica, MCZ 186.081]; Clench \& Turner, 1950: 260, pl. 48, figs 6, 7 .

Pedalion bicolor; Webb, 1936: 32, pl. 3, fig. 11.

Isognomon bicolor; Abbott, 1954: 358; Olsson \& McGinty, 1958: 19; Nowell-Usticke, 1959: 4; Warmke \& Abbott, 1962: 165; Rice \& Kornicker, 1962: 380, pl. 9, figs 10a, 10b; Coomans, 1963: 164; Arnow et al. 1963: 170; Abbott, 1968: 202, fig. 2; Andrews, 1971: 163, fig. na p. 163; Abbott, 1974: 441, pl. 20, fig. 5126; Humfrey, 1975: 218, pl. 24, fig. 10; Emerson \& Jacobson, 1976: 366, pl. 39, fig. 15 (não pl. 44, fig. 4 como indicado); Abbott \& Dance, 1983: 302, fig. na p. 302; Merlano \& Hegedus, 1994: 55, pl. 7, fig. 64.

Perna chemnitziana d'Orbigny, 1846: 346; Schramm, 1867: 22; Schramm, 1869: 22; Dautzenberg, 1900: 230 (syn.).

Pedalion chemnitziana; Webb, 1936: 37, pl. 5, fig. 20.

Isognoma chemnitziana; Clench \& McLean, 1936: 162; Clench \& McLean, 1937: 36.

Melina semiaurita; Dall \& Simpson, 1901: 463 (non Linné, 1758).

Pedalion semiaurita; McLean, 1936b: 117 (non Linné, 1758).

Isognomon (Melina) semiaurita; Aguayo \& Jaume, 1948: 506 (non Linné, 1758).

Isognomon vulsella; McLean, 1951: 22, pl. 4, figs 1, 2 (non Lamarck, 1819).

Isognomon alatus; Tarasconi, 1989: 14 (non Gmelin, 1791: 3339).

Diagnose. Concha com altura maior que o comprimento, fracamente inflada, e com forte ornamentação lamelar. Região do corpo, na face interna das valvas, moderadamente côncava, porém conspícua, com borda nitidamente definida por interrupção abrupta do nácar, formando elevação fraca, arredondada e acentuado desnível que marca a transição para uma região marginal não nacarada, fracamente côncava a plana. Espécimes com extensão máxima de charneira (aproximadamente $18,0 \mathrm{~mm}$ ) apresentam, em média, um sulco ligamentar a cada $1,35 \mathrm{~mm}$ de extensão de charneira $(1: 1,35 \mathrm{~mm})$.

Redescrição. Concha com altura maior que o comprimento, fracamente inflada, obliquamente subquadrada nos espécimes mais regulares (Fig. 1). Contorno muito variável, indo do ovóide ao espatulado, passando pelo mitiliforme, e alongando-se acentuadamente na região ventro-posterior em espécimes vivendo em 
A
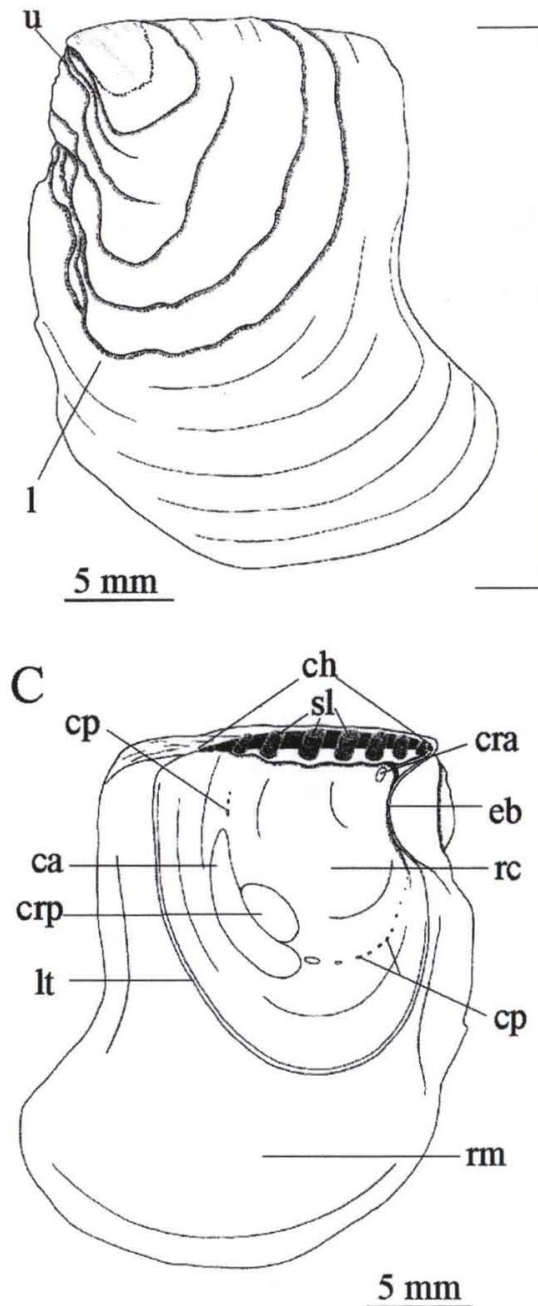

B
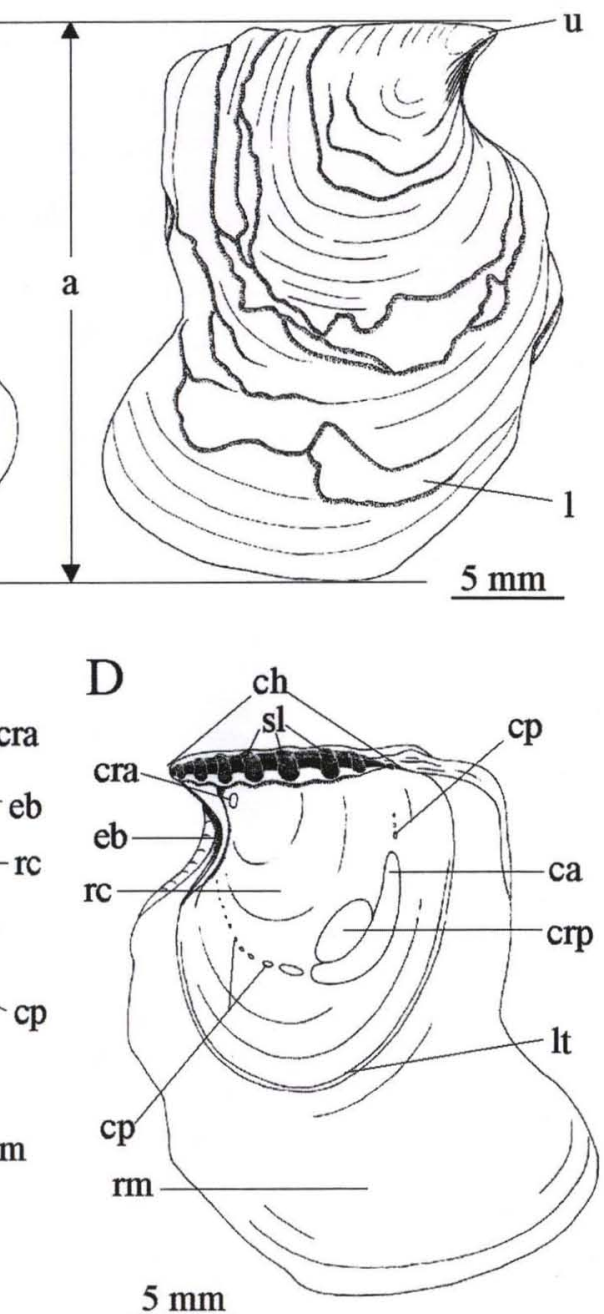

Fig. 1. Isognomon bicolor. (A e B) vista externa e (C e D) vista interna das valvas esquerda e direita, respectivamente. (a) altura, (ca) cicatriz do músculo adutor, (ch) charneira, (cp) cicatrizes dos músculos paliais, (cra, crp) cicatrizes dos músculos retratores anterior e posterior do pé e do bisso, respectivamente, (eb) entalhe bissal, (I) ornamentação lamelar. (It) linha de transição entre a região do corpo e região marginal, ( $\mathrm{rc}$ ) região do corpo, nacarada, (rm) região marginal, não nacarada, (sl) sulcos ligamentais, (u) umbo. 
2

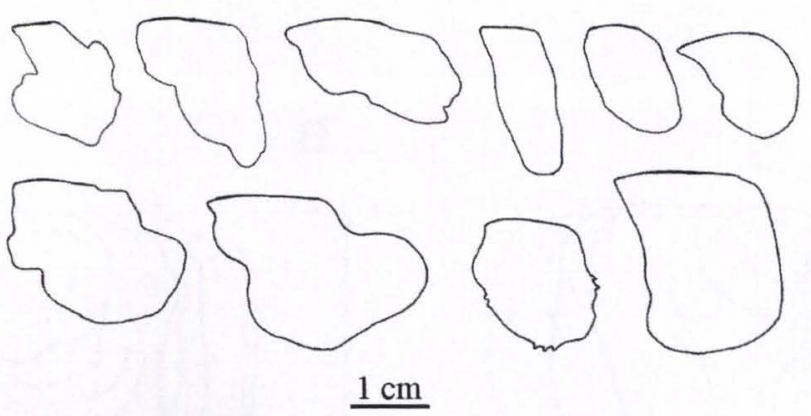

3

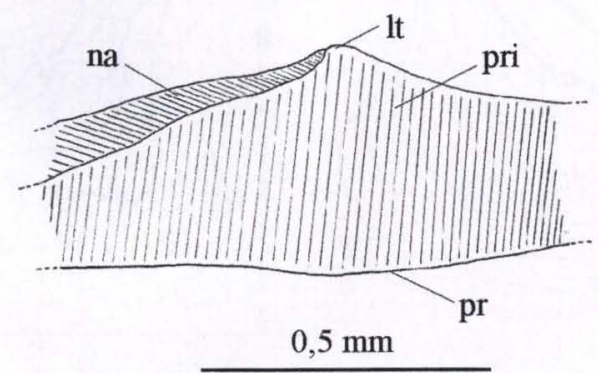

4

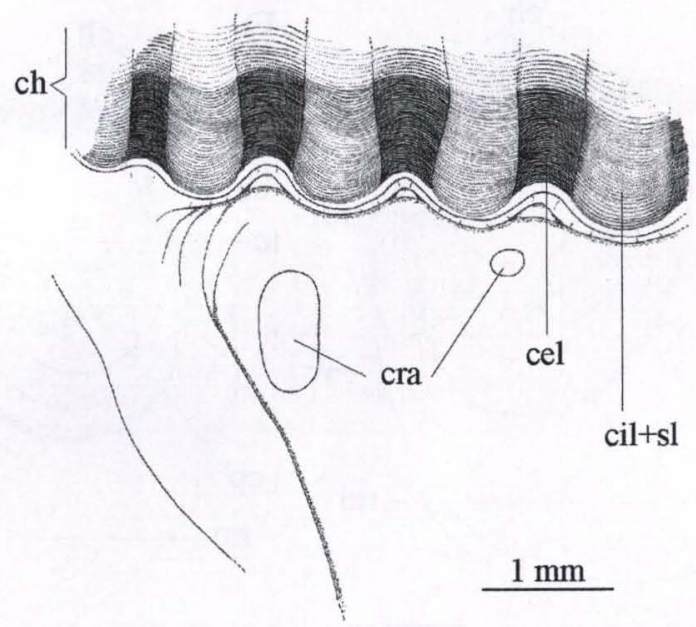

Figs 2-4. Isognomon bicolor. (2) Contorno da concha de 10 espécimes mostrando algumas das variações intraespecíficas. (3) Representação esquemática, e na horizontal, de um segmento de valva cortada segundo um plano dorso-ventral para evidenciar o espessamento que marca a transição abrupta entre a região do corpo e a região marginal. (It) linha de transição, (na) camada nacarada, (pr) perióstraco, (pri) camada prismática. (4) Detalhe da porção anterior e dorsal da valva direita, em vista interna, evidenciando um segmento da charneira e cicatrizes musculares. (cel) camada externa do ligamento, entre sulcos ligamentais adjacentes, (ch) charneira, (cil+sl) camada interna do ligamento no respectivo sulco ligamental, (cra) cicatrizes dos dois músculos retratores anteriores do pé e bisso. 
grandes adensamentos, fendas ou cavidades (Fig. 2). Margem dorsal reta, com umbos (u) na extremidade anterior formando rostro anguloso; margem anterior da região rostral geralmente mais côncava na valva direita, na altura do entalhe bissal (eb); margem ventral arredondada, podendo prolongar-se fortemente, e com maior frequiência, em direção posterior. Valva esquerda ligeiramente mais convexa que a direita; plano de comissura ondulado. Coloração variando do castanho enegrecido, levemente avermelhado, até tons de creme-amarelado ou levemente esverdeado, esmaecendo em direção aos umbos em função do desgaste da concha. Faixas radiais esbranquiçadas, muito tênues, podem estar presentes próximo aos umbos. Valvas normalmente opacas, entretanto translúcidas quando muito finas. Superfície externa ornamentada por lamelas concêntricas (1) irregulares, sobrepostas, quebradiças, conferindo aspecto escamoso à superfície da concha. Formas juvenis com ou sem costelas radiais fracas (or) (Fig. 5), projetando-se para além da margem livre das valvas; costelas erodidas nos indivíduos gerônticos. Perióstraco fino, fortemente aderido, translúcido, freqüentemente erodido nas partes mais velhas da concha.

Face interna das valvas na região que abriga o corpo (rc) apresenta-se moderadamente côncava, revestida por nácar brilhante, de coloração uniforme a iridescente. Borda da região do corpo bem delimitada por interrupção abrupta do nácar, formando elevação fraca, arredondada, e acentuado desnível que marca a transição (lt) para uma região marginal não nacarada, fracamente côncava a plana (rm), constituída apenas pelas camadas prismática e periostracal (Figs 1, 3 e 9). Região marginal estreita a muito expandida, com área menor a bem superior àquela da região do corpo. Cicatrizes do músculo adutor posterior (ca), dos retratores anterior (cra) e posterior (crp) do pé e bisso, e das inserções isoladas dos músculos paliais (cp) restritas à região côncava e nacarada (Figs 1 e 4).

Indivíduos juvenis de 1 a $2 \mathrm{~mm}$ de altura e bem preservados revelam pequeno dente (den) na valva esquerda e correspondente depressão (dep) na direita. Primeiro sulco ligamentar presente em juvenis a partir de $1,3 \mathrm{~mm}$ de altura e $0,9 \mathrm{~mm}$ de extensão de charneira (Figs 7 e 8). Charneira reta (ch), tornando-se edentada por desgaste do dente ao longo do processo de crescimento da concha; área ligamentar achatada, guarnecida com sulcos ligamentais (resilíferos) (sl) paralelos entre si e ao mesmo tempo perpendiculares à linha da charneira. Ligamento multivincular (Figs 1, 4 e 9), com camada externa (cel) depositada por toda a extensão da charneira, exceto nos sulcos ligamentais, onde se deposita a camada interna do ligamento ou resílio (cil). Partes mais velhas e externas do ligamento freqüentemente fraturadas. Valyas unidas através da porção do resílio e da camada externa do ligamento de deposição mais recente, ambas localizadas ventralmente à área fraturada. Sulcos ligamentais aumentando em número com o crescimento da concha; espécimes com extensão máxima de charneira (aproximadamente 18,0 $\mathrm{mm}$ ) apresentam, em média, um sulco ligamentar a cada 1,35 mm de extensão de charneira $(1: 1,35 \mathrm{~mm})$.

Prodissoconcha. Prodissoconcha (Fig. 6) de contorno triangular, com umbos pontiagudos subcentrais; margens anterior e posterior uniformemente arredondadas, margem ventral convexa. Superfície externa lisa, a não ser pela presença de linhas de crescimento concêntricas, mais evidentes próximo às margens. Dimensões: $200 \mu \mathrm{m}$ de altura por $225 \mu \mathrm{m}$ de comprimento. 


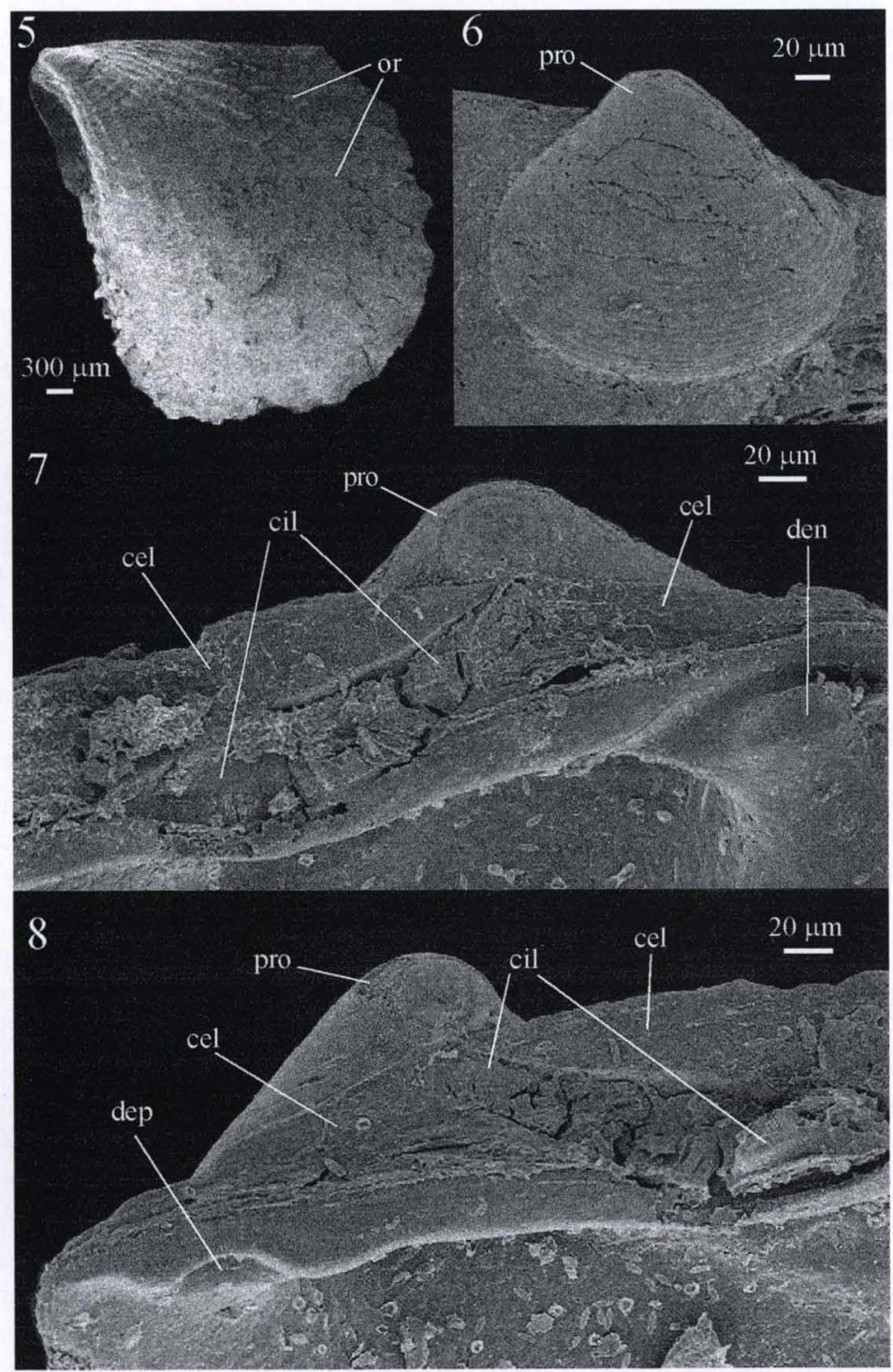

Figs 5-8. Isognomon bicolor. (5) Vista externa da valva esquerda de espécime juvenil, evidenciando costelas radiais fracas. (or) ornamentação radial. (6) Vista externa da valva esquerda da prodissoconcha de um dos raros espécimes coletados com a concha nepiônica relativamente bem preservada. (pro) prodissoconcha. $(7-8)$ Vista interna das valvas esquerda e direita, respectivamente, de espécime juvenil, evidenciando detalhes da charneira raramente preservados durante o crescimento. (cel) camada externa do ligamento, (cil) camada interna do ligamento (primeiro resílio a se formar), (den) dente, (dep) depressão de encaixe do dente da valva oposta, (pro) prodissoconcha. 
9

A
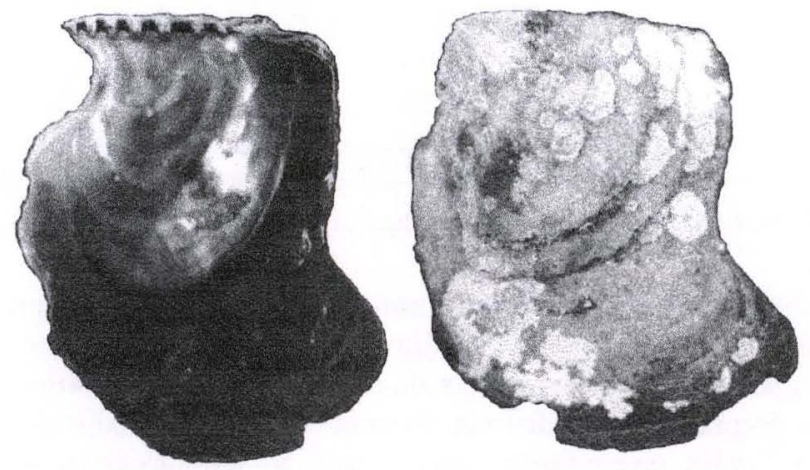

$5 \mathrm{~mm}$

\section{B}

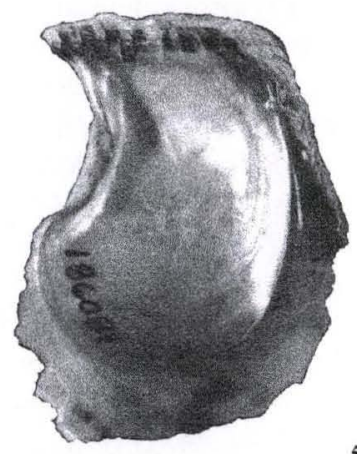

$5 \mathrm{~mm}$

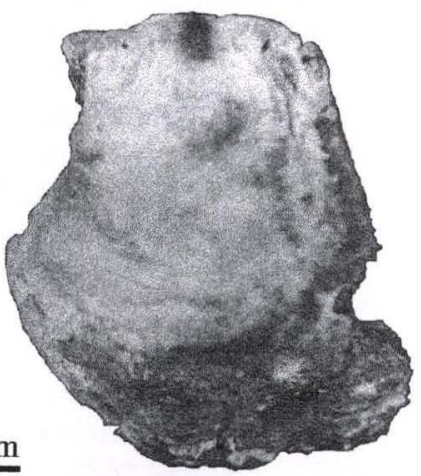

C

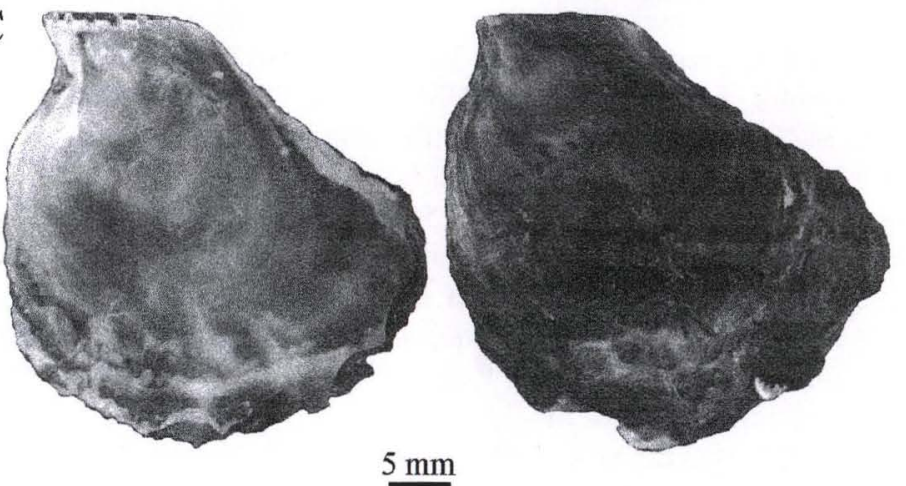

Fig. 9. Da esquerda para a direita, vista interna da valva direita e vista externa da valva esquerda de: (A) forma regular de Isognomon bicolor, (B) lectótipo de Isognomon bicolor (C.B. Adams, 1845) [Jamaica, MCZ 186081]; (C) síntipo de Isognomon alatus (Gmelin, 1791) ["West Indies", ZMUC-BIV 387]. 
Dados morfométricos da dissoconcha [mínimos (espécimes com o primeiro sulco ligamental) - máximos]. Altura (mm): 1,3-36,0; comprimento da charneira (mm): 0,9-17,5; sulcos ligamentais: 1-13.

Distribuição geográfica. BERMUDAS; E.U.A., Flórida, Texas; BAHAMAS; MÉxico; CubA; JAMAiCA; PorTo Rico; IlHas Virgens, Saint Thomas; MARTinICA; TRInIDAD; Panamá; Colômbia, Santa Marta; Venezuela, Ilha Margarita; BRASIL, Rio Grande do Norte, Pernambuco, Bahia, Rio de Janeiro, São Paulo, Santa Catarina.

Habitat. Poças de maré no supralitoral até sete metros de profundidade no infralitoral. Costões amplos no mesolitoral, banhados por ondas de baixo impacto e correndo subparalelas à superfície da rocha apresentam indivíduos densamente agregados, formando faixa distinta. Presente em número reduzido no interior de depressões, fendas, ou cavidades abandonadas por animais do mesolitoral, na face protegida de rochas e de costões sujeitos a fortes ondas.

Material examinado. Isognomon bicolor. (*) lotes coletados pelos autores, e (+) lotes recebidos por doação de JCT, depositados pelos autores. BERMUDAS, 1 valva direita (v. d.), MORG 27.012. E.U.A., Florida, 16 conchas +1 valva esquerda (v. e.), MORG 25.332, Monroe County, 25 espécimes, MZUSP 32.550. JAMAiCA, MCZ 186.081 (lectótipo). PORTO RiCo/MARTINICA, 6 conchas, MZUSP 32.551. ILHAS VIRGENS, Saint Thomas, 3 conchas, MZUSP 11.281. VENEZUELA, Isla Margarita, 12 espécimes, MZUSP 32.552. TRINIDAD, 2 conchas, MZUSP 11.282. BRASIL, Rio Grande do Norte, Natal, Praia da Ponta Negra, 3 espécimes, MZUSP 32.553*; Pernambuco, Recife, Praia da Boa Viagem, 5 espécimes, MZUSP 32.554*; Bahia, Salvador, Praia de Itapuã, 1 concha, L.T.; Rio de Janeiro, Arraial do Cabo, Prainha, 70 espécimes, MZUSP 32.555*, Enseada do Forno, Ponta da Fortaleza, 15 espécimes, MZUSP 32.556*, Niterói, Praia de Itaipu, 97 espécimes, MZUSP 32.557*; São Paulo, Ubatuba, Prainha do Matarazzo, 73 espécimes, MZUSP 32.558*, Praia do Tenório, 9 espécimes, MZUSP 32.559*, Praia do Lázaro, 63 espécimes, MZUSP 32.560*, Ilha Bela, Praia de Siriuba, 6 espécimes, MZUSP 32.561*, Praia da Barra Velha, 13 espécimes, MZUSP 32.562*, São Sebastião, Praia de São Francisco, 5 espécimes, MZUSP 32.563*, Ponta do Baleeiro, 6 espécimes, MZUSP 32.564*, Praia de Barequeçaba, 7 espécimes, MZUSP 32.565*, 2 espécimes, MZUSP 32.566*, 94 espécimes, MZUSP 32.567*, 7 espécimes, MZUSP 32.568*, 14 espécimes, AMNH 293968*, Praia de Guaecá, 8 espécimes, MZUSP 32.569*, Praia de Toque-toque Grande, 11 espécimes, MZUSP 32.570*, Bertioga, Praia de Itaguaré, 5 espécimes, MZUSP 32.571*, Guarujá, Praia de Pernambuco, Ilha do Mar Casado, 5 espécimes, MZUSP 32.572*, Praia do Obuseiro, 39 espécimes, MZUSP 32.573*, Ilha da Moela, 89 espécimes, MZUSP 32.574*, São Vicente, Praia de Itararé, Ilha Porchat, 3 espécimes, MZUSP 32.575*; Santa Catarina, Itapema, Canto da Praia, 1 concha, J.C.T., 7 espécimes, MZUSP 32.576+, Porto Belo, Enseada da Caixa D'Aço, 2 espécimes, MZUSP 32.577+, Bombinhas, Praia dos Ingleses, 7 espécimes, MZUSP 32.578+, Praia da Sepultura, 2 espécimes, MZUSP 32.579+, Enseada de Zimbros, 3 espécimes, MZUSP 32.580+, Florianópolis, Praia da Joaquina, 54 espécimes, MZUSP 32.581+. 


\section{Isognomon alatus (Gmelin, 1791)}

Fig. $9 \mathrm{C}$

Ostrea alata Gmelin, 1791: 3339.

Isognomum alatum; Poulsen, 1878: 16.

Perna alata; Clessin, 1891: 27, pl. 11, fig. 3; Lamy, 1929: 202.

Perna alatus; Rush, 1891: 70.

Melina alata; Dall \& Simpson, 1901: 462; Thiele, 1910: 126; Salisbury, 1953: 51.

Isognoma alata; Clench \& McLean, 1936: 162; McLean, 1936a: 40; Clench \& McLean, 1937: 35, pl. 6, figs 1 e 2 .

Pedalion alata; McLean, 1936b: 117; Webb, 1936: 19, fig. p.19; Morris, 1951: 20, pl. 9, fig. 1; Smith \& Baily, 1953: 92, fig. 1267; Vilas \& Vilas, 1983: 27, pl. 1, fig. 9.

Isognomon (Melina) alata; Aguayo \& Jaume, 1948: $\mathrm{n}^{\circ} 506$.

Isognomon alata; McLean, 1951: 22, pl.4, fig.3; Nowell-Usticke, 1957: 115.

Isognomon alatus; Abbott, 1954: 358, pl. 35, fig. b; Olsson \& McGinty, 1958: 19; Nowell-Usticke, 1959: 4; Warmke \& Abbott, 1962: 165, pl. 32, fig. c; Rice \& Kornicker, 1962: 379, pl. 9, figs 11a e 11b; Abbott, 1968: 202, fig. 1; Houbrick, 1968: 18; Work, 1969: 686; Rios, 1970: 161; Andrews, 1971: 162, fig. na p.163; Abbott, 1974: 441, pl. 20, fig. 5124; Rios, 1975: 203, pl. 65, fig. 985; Humfrey, 1975: 218, pl. 24, fig. 11; Morris, 1975: 24, pl. 13, fig. 17; Emerson \& Jacobson, 1976: 365, pl.44, fig. 3; Abbott \& Dance, 1983: 302, fig. na p.302; Rios, 1985: 220, pl. 79, fig. 1108; Sabeli, 1985: 416, pl. 314; Cosel, 1986: 112, fig. 29; Rios, 1994: 241, pl. 83, fig. 1185; Merlano \& Hegedus, 1994: 54, pl. 7, fig. 62; Abbott \& Morris, 1995: 21, pl. 21, fig. 7.

Isognomum alata; Moore, 1958: 126 e 128.

Perna obliqua Lamarck, 1819: 140; Deshayes \& Edwards 1836: 75 (syn.); Anton, 1839: 17; Hanley, 1843: 258; d'Orbigny, 1846: 346; Beau, 1851: 426; Schramm, 1867: 22; Schramm, 1869: 22; Dall, 1889: 36; Maury, 1912: 41, pl. 7, fig. 6; Lamy, 1933: 393.

Melina obliqua; Maury, 1920: 55.

Isognomon obliquum; Peile, 1926: 92.

Perna ephippium; Reeve, 1858: pl. 2, fig. 8 (non Linné, 1758).

Material examinado. Isognomon alatus. E.U.A., Florida, 2 v. d. + 2 v. e., MORG 22.505, 1 concha, MORG 24.261, 5 conchas, MZUSP 32.582; Miami, Mathewson Hammock, 4 conchas +1 v. d., BMSM 1.005, Little Duck Key, 3 conchas, BMSM 1.004, Marathow Key, 1 concha, MNRJ-HSL 569, Monroe County, 6 espécimes, MZUSP 32.583. BAHAMAS, 2 conchas, MZUSP 11.275, 1 concha, MORG 515. MÉXICO, 1 concha, MORG 35.741. "WEST INDIES", 1 concha, ZMUC-BIV 387 (síntipo). JAMAICA, Kingston, 1 concha +4 v. d. + 1 v. e., MNRJ-HSL 1.334. IlHAS VIRGENS, Saint Thomas, 1 concha, MZUSP 11.276, 1 concha, MZUSP 11.279. ANTIGUA, English Harbour, 5 conchas, BMSM 1.003.

De todas as localidades visitadas no litoral do Rio Grande do Norte, Pernambuco, Bahia, Rio de Janeiro, São Paulo, Santa Catarina e Rio Grande do Sul, espécimes do gênero Isognomon só não foram encontrados neste último Estado. Também não se verificou a ocorrência de espécies do gênero Isognomon no Arquipélago de Fernando de Noronha, como ocorrera para MATTHEWS \& KEMPF (1970) ao fazerem o levantamento de moluscos dessa localidade.

A análise dos caracteres conquiliológicos dos representantes do gênero Isognomon provenientes das localidades brasileiras visitadas, comparativamente ao lectótipo de I. bicolor (JAMAICA, MCZ 186.081), ao síntipo de I. alatus (Gmelin, 1791) ("WEST INDIES", ZMUC-BIV 387), e às respectivas descrições originais, 
demonstrou que todos os espécimes, sem exceção, são conspecíficos com I. bicolor. Obteve-se igual resultado na análise de todos espécimes do gênero Isognomon provenientes do litoral brasileiro, cedidos para o presente estudo pelos conquiliologistas e pesquisadores.

Isognomon bicolor (C.B. Adams, 1845) e I. alatus (Gmelin, 1791) são espécies similares (ABBOTT 1974; HUMFREY 1975) pelos caracteres externos da concha, o que dificulta a distinção interespecífica de espécimes na mesma faixa de tamanho. Essa dificuldade aumenta quando ocorrem distorções na concha decorrentes da forte competição intraespecífica pelo espaço, ou da ocupação de fendas e cavidades no substrato.

Isognomon bicolor tem menor altura, a qual não atinge a metade da altura máxima $(90 \mathrm{~mm}$ ) referida na literatura (SIUNG 1980) para I. alatus. Principal caráter distintivo de I. bicolor é a borda da região do corpo, na face interna das valvas, nitidamente definida por interrupção abrupta do nácar, formando elevação fraca, arredondada, e desnível acentuado na transição para a região marginal não nacarada. MCLEAN (1951) e EMERSON \& JACOBSON (1976) são os únicos a destacar a importância desse caráter em I. bicolor, ausente em I. alatus. Esta última espécie caracteriza-se pela concha excepcionalmente comprimida e sulcos ligamentais retangular-oblongos (EMERSON \& JACOBSON 1976).

A avaliação da importância da relação entre o "número de sulcos ligamentais versus extensão da charneira" para a distinção entre $I$. bicolor e $I$. alatus ficou parcialmente prejudicada pela dificuldade de obtenção de maior número de exemplares e de série de crescimento completa, particularmente da última espécie (Tab. I). Entretanto, os elementos da tabela I permitem identificar que I. bicolor tem maior número de sulcos por unidade de extensão de charneira que $I$. alatus, a primeira espécie atingindo 13 sulcos nos maiores exemplares da amostra com 17,1-18,0 mm de extensão de charneira, número esse só alcançado por exemplares de I. alatus com charneira de 30,1-31,0 mm de extensão. Essa mesma tabela permite identificar que nas classes de extensão de charneira superiores a $10,0 \mathrm{~mm}$, nas quais $I$. alatus está melhor representada, o número mínimo de sulcos em I. bicolor é superior ao número máximo em I. alatus. Contudo, essa fronteira mínimo-máximo é muito próxima ou pode mesmo se sobrepor em alguns espécimes de uma e outra espécie. A possibilidade dessa justaposição do caráter para ambas espécies, em indivíduos na mesma faixa de tamanho, requer que a identificação seja complementada pela análise dos demais caracteres conquiliológicos.

Aos caracteres diagnósticos para I. bicolor, pode-se considerar a maior proximidade entre os sulcos ligamentais. Estes distam, em média, de 1,35 mm $(1: 1,35 \mathrm{~mm})$ nos maiores espécimes com aproximadamente $18 \mathrm{~mm}$ de extensão de charneira. Em I. alatus com essa mesma dimensão de charneira a distância média entre os sulcos é de aproximadamente $2,20 \mathrm{~mm}(1: 2,20 \mathrm{~mm})$, alcançando no maior exemplar examinado (charneira com 40,5 mm de comprimento) (Tab. I) uma relação de $1: 2,38 \mathrm{~mm}$.

Isognomon bicolor corresponde a novo registro para o Brasil, com instalação recente na região de São Sebastião, e por toda região sudeste e parte da região sul do País. Essa instalação ocorreu, muito provavelmente, entre as décadas de setenta 
e oitenta do século recém findo, como se deduz dos trabalhos de TARASCONI (1989) e MigotTo et al. (1993). A expansão populacional da espécie deve ter ocorrido durante a transição para a década de noventa, fato que motivou o alerta aos autores do presente estudo sobre a invasão dos costões de São Sebastião por Isognomon sp., disputando espaço e reduzindo drasticamente a presença antes maciça dos bivalves autóctones Brachidontes e ostreídeos.

Tabela I. Variação do número de sulcos ligamentais com o crescimento da charneira.

\begin{tabular}{|c|c|c|c|c|}
\hline \multirow{2}{*}{$\begin{array}{l}\text { Classes de comprimento } \\
\text { de charneira }(\mathrm{mm})\end{array}$} & \multicolumn{2}{|c|}{ Isognomon bicolor } & \multicolumn{2}{|c|}{ Isognomon alatus } \\
\hline & Número de sulcos & Número de indivíduos & Número de sulcos & Número de individuos \\
\hline $0,1-1,0$ & 1 & 1 & - & - \\
\hline $1,1-2,0$ & 2 & 1 & - & - \\
\hline $2,1-3,0$ & 2 & 1 & - & - \\
\hline $3,1-4,0$ & 3 & 3 & - & - \\
\hline $4,1-5,0$ & $3-4$ & 7 & - & - \\
\hline $5,1-6,0$ & $4-5$ & 2 & - & - \\
\hline $6,1-7,0$ & $4-6$ & 4 & 3 & 1 \\
\hline $7,1-8,0$ & $5-6$ & 5 & 4 & 1 \\
\hline $8,1-9,0$ & $6-7$ & 7 & - & - \\
\hline $9,1-10,0$ & $6-7$ & 8 & $5-6$ & 3 \\
\hline $10,1-11,0$ & $7-9$ & 11 & 5 & 1 \\
\hline $11,1-12,0$ & $7-10$ & 9 & 6 & 2 \\
\hline $12,1-13,0$ & $8-10$ & 10 & $5-7$ & 5 \\
\hline $13,1-14,0$ & $8-11$ & 7 & 7 & 1 \\
\hline $14,1-15,0$ & $8-11$ & 6 & 7 & 4 \\
\hline $15,1-16,0$ & $9-11$ & 3 & $7-8$ & 3 \\
\hline $16,1-17,0$ & 10 & 1 & $7-10$ & 4 \\
\hline $17,1-18,0^{*}$ & 13 & 1 & 8 & 1 \\
\hline $18,1-19,0$ & - & - & $8-9$ & 4 \\
\hline $19,1-20,0$ & - & - & $8-10$ & 5 \\
\hline $20,1-21,0$ & - & - & 8 & 1 \\
\hline $21,1-22,0$ & - & - & - & - \\
\hline $22,1-23,0$ & - & - & $9-11$ & 2 \\
\hline $23,1-24,0$ & - & - & $9-10$ & 2 \\
\hline $24,1-25,0$ & - & - & $9-12$ & 2 \\
\hline $25,1-26,0$ & - & - & $10-11$ & 2 \\
\hline $26,1-27,0$ & - & - & $10-12$ & 3 \\
\hline $27,1-28,0$ & - & - & - & - \\
\hline $28,1-29,0$ & - & - & - & - \\
\hline $29,1-30,0$ & - & - & 12 & 1 \\
\hline $30,1-31,0$ & - & - & 13 & 1 \\
\hline $40,1-41,0^{\star *}$ & - & - & 17 & 1 \\
\hline Total & & 87 & & 50 \\
\hline
\end{tabular}

(*) Isognomon bicolor, $\left(^{* *}\right)$ Isognomon alatus: maior comprimento de charneira na amostra examinada. (-) ausência de dados.

As previsões de instalação de I. bicolor nas regiões sudeste e sul do Brasil, no decorrer das décadas sugeridas, baseiam-se no fato de que o gênero Isognomon não é referido para São Sebastião desde o levantamento malacológico pioneiro por IHERING (1897), até o mais recente realizado de VI/1982 a II/1983 e publicado por MigotTo et al. (1993). Neste último trabalho os autores complementam o levantamento que realizaram compilando toda bibliografia precedente que aborda a mala- 
cofauna dessa localidade. Essa bibliografia compilada também não faz referência ao gênero Isognomon. Outro referencial importante é o fato do registro pioneiro do gênero Isognomon para a região sul do Brasil ter sido feito por TARASCONI (1989) a partir de uma única concha encontrada em julho de 1984 no "cascalho intertidal" da praia de Itapema, Santa Catarina. Esse material testemunho, identificado por TARASCONI (1989) como "I. alatus Gmelin, 1791" (sic) foi analisado pelos autores do presente estudo que constataram um equívoco de identificação, sendo o espécime conspecífico com I. bicolor.

Como todo material examinado e pertencente a I. alatus é proveniente exclusivamente de localidades fora do Brasil, e todo material coletado no litoral brasileiro pelos autores ou colaboradores é conspecífico com I. bicolor, os fatos sugerem duas hipóteses: a de que a espécie $I$. alatus seja extremamente rara no Brasil, ou a de que $I$. bicolor, com distribuição ampla pelo litoral brasileiro, como comprovado neste estudo, tenha sido identificada equivocadamente como $I$. alatus, gerando igualmente os registros equivocados para as regiões sudeste e sul. A variabilidade intraespecífica em ambas espécies, a ocorrência de similaridades na forma entre elas, mais o fato de compartilharem um hábito de vida e habitats similares, favorecem esses equívocos. $\mathrm{O}$ não encontro do material-testemunho, e a falta de indicação por RIOS (1994) da instituição depositária dos espécimes que lhe serviram para o registro de I. alatus na região sudeste, não permitiram a análise necessária desse material para a verificação da hipótese de equívoco na identificação.

Isognomon radiatus é outra espécie que demanda mais esforços de busca para se comprovar o registro feito para o Brasil por Аввотт (1974), Аввотт \& DANCE (1983) e ABBOTT \& MORRIS (1995), que não mencionam localidades precisas e fonte do registro.

AGRADECIMENTOS. À FAPESP e ao CNPq, pelo suporte financeiro. Aos seguintes pesquisadores e respectivas instituições por autorizarem o exame direto no acervo, ou o empréstimo de material científico: Dr. J.L.M. Leme (MZUSP), Dra. N.C. Salgado (MNRJ), Dra. I. Swoboda (MORG), Dr. J.H.N. Leal (BMSM), Dr. T. Schiotte (ZMUC) e Dr. A. Baldinger (MCZ). À Dra. P. Mikkelsen e ao Dr. J. Cordeiro (AMNH), pela coleta e doação, a nosso pedido, das três espécies de Isognomonidae da Flórida. À Dra. H. Matthews-Cascon (UFCE), por informar sobre o extravio do material original de "Isognomon cf. alatus", coligido por MATTHEWS \& KEMPF (1970) no Atol das Rocas. A J.C. Tarasconi, do Rio Grande do Sul, e L. Trinchão, da Bahia, pelo empréstimo de Isognomon de suas coleções particulares. A J. Coltro Júnior, de São Paulo, pela doação de conchas de Isognomonidae. Aos colegas pesquisadores C. Magenta, C. Miyaji, F.R. Robles, L.R.L. de Simone e P.J.S. de Souza Jr por coletarem, a nosso pedido, espécimes de Isognomonidae em suas viagens pelo litoral brasileiro. Ao Dr. J.C. de Freitas, Diretor do CEBIMar/USP, por autorizar o uso da infra-estrutura da instituição. À técnica L. M. Guimarães, laboratório de MEV-MZUSP, pelas imagens através do microscópio eletrônico de varredura. Ao IBAMA, por autorizar a visita e coletas no Parque Nacional Marinho do Arquipélago de Fernando de Noronha. Ao Dr. J.L.M. Leme pela leitura crítica do texto e sugestões, ao Dr. U.R.M. de Souza pela assessoria na parte sistemática, e aos consultores Dra. L.M.Z. Richinitti e Dr. J.W. Thomé pelas valiosas sugestões para o aprimoramento do texto. 


\section{REFERÊNCIAS BIBLIOGRÁFICAS}

Аввотт, R.T. 1954. American seashells. New Jersey, D. Van Nostrand Company, 541p.

1968. Seashells of North America: a guide to field identification. New York, Golden

Press, 280p.

1974. American seashells. 2. ed. New York, Van Nostrand Reinhold Company, 663p.

Aвbott, R.T. \& S.P. DANCE. 1983. Compendium of seashells. New York, E.P. Dutton, $2^{\text {nd }}$ ed., 410p.

Аввотт, R.T. \& P.A. Morris. 1995. A field guide to shells - Atlantic and Gulf coasts and the West

Indies. New York, Houghton Mifflin Company, $4^{\text {th }}$ ed., 350p.

ADAMS, C.B. 1845. Specierum novarum conchyliorum, in Jamaica Repertorum, Synopsis. Proc. Boston

Soc. Nat. Hist. 2: 1-17.

Aguayo, C.G. \& M.L. Jaume. 1947-1951. Catálogo moluscos de Cuba. La Habana, 725p.

AndRews, J. 1971. Sea shells of the Texas coast. Austin, Univ. Texas Press, 298p.

ANTON, H.E. 1839. Verzeichniss der Conchylien welche sich in der Sammlung von Hermann

Eduard Anton befinden. Halle, Eduard Anton, 110p.

Arnow, L.; F. ST. Clair, \& T. Arnow. 1963. The Mollusca of a lagoonal area at Playa de Vega Baja,

Puerto Rico. Carib. Jour. Sc. 3 (43): 163-172.

BEAU, M. 1851. Catalogue des coquilles trouvées à l'île de la Guadeloupe. Jour. Conch., Paris, 2: 422-431.

Boss, K.J. 1982. Mollusca, p. 945-1166. In: S.P. PARKER (Ed.). Synopsis and classification of living organisms. New York, McGraw Hill Book Company, Vol. 1, 1166p.

CLENCH, W.J. \& R.A. MCLEAN. 1936. Marine bivalves collected by the Harvard-Bahama Expedition of 1935. Mems Soc. Cubana Hist. Nat. "Felipe Poey” 10 (3): 157-168.

CLENCH, W.J. \& R.A. MCLEAN. 1937. Marine bivalves from little and great Abaco, Grand Bahama, and Eleuthera Islands. Mems Soc. Cubana Hist. Nat. "Felipe Poey” 11 (1): 31-42.

Clench, W.J. \& R.D. TuRner. 1950. The Western Atlantic marine mollusks described by C.B. Adams. Occ. Pap. Mollusks Harv. 1 (15): 233-403.

Clessin, S. 1891. Malleacea, p. 26-51. In: F.H.W. Martini \& J.H. Chemnitz (Ed.). Systematisches Conchylien-Cabinet, Nürnberg, Bauer und Raspe, Vol. 8, no. 1, 108p.

COAN, E.V.; P.V. SCOTT \& F.R. BERnARD. 2000. Bivalve seashells of Western North America. Santa Barbara, Santa Barbara Museum of Natural History, 764p.

Coomans, H.E. 1963. The marine Mollusca of Saint Martin, Lesser Antilles, especially the French part. Jour. Conch., Paris, 103: 113-186.

Cosel, R. von. 1986. Moluscos de la region de la Cienaga Grande de Santa Marta (costa del caribe de Colombia). An. Inst. Invest. Mar. Punta de Betin 15-16: 79-370.

DALL, W.H. 1889. A preliminary catalogue of the shell-bearing marine mollusks and brachiopods of the southeastern coast of the United States, with illustrations of many of the species. Bull. U.S. Nat. Mus. 37: 1-221.

Dall, W.H. \& C.T. Simpson. 1901. The Mollusca of Porto Rico. U.S. Fish Comm. Bull. 1: 351-524.

DESHAYES, G. P. \& H.M. EDWARDS. 1836. Histoire naturelle des animaux sans vertèbres, par J.B.P.A. de M. de Lamarck. Paris, J.B. Baillière, 2 éd., vol. 7, 735p.

Dautzenberg, P. 1900. Croisères du Yacht Chazalie dans l'Atlantique. Mollusques. Mem. Soc. Zool. Fr. 13: $145-265$.

D’Orbigny, A.D. 1846. Mollusques, vol. 2, p. 203-368. In: R. DE LA Sagra. Histoire Physique, Politique et Naturelle de L'Ile de Cuba. Paris, A. Bertrand, 1841-1853, Vol. 2, 380p, 28 pls.

EMERSON, W.K. \& M.K. JACOBSON. 1976. The American Museum of Natural History. Guide to shells. Land, freshwater and marine, from Nova Scotia to Florida. New York, Alfred A. Knopf Inc., $482 p$.

GMELIM, J.F. 1791. Ostrea alata, p. 3339. In: C. LINNÉ (Ed.) Systema naturae per regna tria naturae:

13 ed. aucta, reformata, cura J.F. Gmelin. Lipsiae, G.E. Beer, Vol. 1, pt. 6, p. 3021-3910.

HANLEY, S.C.T. 1842-1856. An illustrated and descriptive catalogue of recent bivalve shells. London, $392 \mathrm{p}$. 
HoubRiCK, J.R. 1968. A survey of the littoral marine mollusks of the Caribbean coast of Costa Rica. Veliger 11 (1): 4-23.

Humfrey, M. 1975. Sea shells of the West Indies. New York, Taplinger Publishing Co., 351p. IHeRING, H. von. 1897. A ilha de São Sebastião. Revta Mus. Paulista 2: 129-171.

LAMARCK, J.B.P.A. DE M. DE. 1819. Histoire Naturelle des Animaux sans Vertèbres. Paris, Verdiere, Vol. 6, 343p.

Lamy, M.E. 1929. Les Malleacea de la Mer Rouge. Bull. Mus. Nat. Hist. Nat. 2, sér. 1: 150-156.

. 1933. Notes sur les espèces Lamarckiennes du genre Perna Bruguière. 1792. Bull. Mus. Nat. Hist. Nat. 2, Sér. 5: 393-399.

MATTHEWS, H.R. \& M. KEMPF. 1970. Moluscos marinhos do norte e nordeste do Brasil. II - Moluscos do Arquipélago de Fernando de Noronha (com algumas referências ao Atol das Rocas). Arq. Ciênc. Mar 10 (1): 1-53.

MAURY, C.J. 1912. A contribution to the paleontology of Trinidad. Jour. Acad. Nat. Sc. Philadelphia, 2 ser., 15: 23-112.

. 1920. Recent mollusks of the Gulf of Mexico and Pleistocene and Pliocene species from the Gulf States. Part 1. Bull. Amer. Paleont. 8 (34): 1-115.

McLean, R.A. 1936a. A list of bivalves from Northern Cuba. Mem. Soc. Cubana Hist. Nat. "Felipe Poey" 10 (1): 39-42.

. 1936b. Some marine bivalves from the Bahama Islands. Nautilus 49 (4): 116-119.

. 1951. The pelecypoda or bivalve mollusks of Porto Rico and the Virgin Islands. Scient.

Surv. Puerto Rico 17 (1): 1-183.

Merlano, J.M.D. \& M.P. Hegedus. 1994. Moluscos del caribe colombiano - Un catálogo ilustrado.

Santafé de Bogotá, Fundación Natura, Invemar, 291p.

Migotto, A.E.; C.G. TiAgo \& A.R.M. MagAlhÃES. 1993. Malacofauna marinha da região costeira do

Canal de São Sebastião, SP, Brasil: Gastropoda, Bivalvia, Polyplacophora e Scaphopoda. Bol. Inst.

Oceanográfico, São Paulo, 41 (1/2): 13-27.

MoORE, D.R. 1958. Additions to Texas marine Mollusca. Nautilus 71 (4): 124-129.

Morris, P.A. 1951. A field guide to the shells of our Atlantic and Gulf coasts. Boston, Houghton Mifflin Co., $2^{\text {nd }}$ ed., $236 \mathrm{p}$.

- 1975. A field guide to shells of the Atlantic and Gulf coasts and the West Indies. Boston, Houghton Mifflin Co., $3^{\text {rd }}$ ed.,330p.

Nowell-Usticke, G.W. 1957. Marine shells from Altona Lagoon, St. Croix, Virgin Islands. Nautilus 70 (4): 113-116.

. 1959. A check list of the marine shells of St. Croix, U.S. Virgin Islands with random annotations. Privately printed, 90p.

Olsson, A.A. \& T.L. McGinTy. 1958. Recent marine molluscs from the Caribbean coast of Panama with the description of some new genera and species. Bull. Amer. Paleont. 39 (177): 1-58.

PeILE, A.J. 1926. The Mollusca of Bermuda. Proc. Malac. Soc. London 17: 71-98.

PoulsEn, C.M. 1878. Catalogue of West Indian shells in the collection of C.M. Poulsen. Copenhagen, $16 \mathrm{p}$.

ReEve, L.A. 1858. Monograph of the genus Perna. In: Conchologia Iconica: or, illustrations of the shells of molluscous animals. London, Lovell Reeve, 1859, Vol. 11.

Rice, W.H. \& L.S. Kornicker. 1962. Mollusks of Alacran Reef, Campeche Bank, Mexico. Publs Inst. Mar. Sci. Univ. Texas 8: 366-403.

Rıos, E.C. 1970. Coastal Brazilian seashells. Rio Grande, Fundação Cidade do Rio Grande, Museu Oceanográfico de Rio Grande, 255p.

- 1975. Brazilian marine mollusks iconography. Rio Grande, Fundação Universidade de Rio Grande, 331p.

1985. Seashells of Brazil. Rio Grande, Fundação Universidade do Rio Grande, 328p.

1994. Seashells of Brazil, 2. ed. Rio Grande, Fundação Universidade do Rio Grande, 368p.

RusH, W.H. 1891. List of species collected on the Islands of St. Thomas, St. Kitts, Barbados, Jamaica 
and at Pensacola, Florida, with prefatory notes. Nautilus 5 (5): 65-70.

SABELI, B. 1985. Conchiglie, 3. ed. Milano, Arnoldo Mondadori Editore, 512 p.

SAlisbury, A.E. 1953. Mollusca of the University of Oxford Expedition to the Cayman Islands 1938. Proc. Malac. Soc. London 30: 39-54.

Schramm, A. 1867. Catalogue des coquiles et des crustacés de la Guadeloupe, envoyés a l'Exposition universalle de $\mathbf{1 8 6 7}$ par l'administration de la Colonie. Collections Caillet et I. Desbonne. Determinations et classements de A. Schramm. Basse-Terre, Imprimerie du Gouvernement, $27 \mathrm{p}$.

- 1869. Catalogue des coquiles et des crustacés de la Guadeloupe, envoyés a l'Exposition universalle de 1867 par l'administration de la Colonie. Collections Caillet et I. Desbonne. Determinations et classements de A. Schramm. Basse-Terre, Imprimerie du Gouvernement, 2 ed., $27 \mathrm{p}$.

SIUNG, A.M. 1980. Studies on the biology of Isognomon alatus Gmelin (Bivalvia: Isognomonidae) with notes on its potential as a commercial species. Bull. Mar. Sci. 30 (1): 90-101.

SMITH, M. \& J.L. BAILY. 1953. World-wide sea shells; illustrations, geographical range and other data covering more than sixteen hundred species and sub-species of molluscs,by Maxwell Smith, together with two articles by Dr. Joshua L. Baily. Windermere, Tropical Laboratory, $139 \mathrm{p}$.

TARAsConi, J.C. 1989. Novos registros de moluscos marinhos para o litoral de Santa Catarina. Inf. Soc. Brasil. Malacol. 96: 12-16.

THIELE, J. 1910. Molluskenfauna West-indies. Zool. jahrb. Jena (Suppl. 11): 109-132.

Trueman, E.R. 1954. The structure of the ligament of Pedalion (Perna). Proc. Malacol. Soc. London 30 (6): 160-166.

VILAS, C.N. \& N.R. VILAS. 1983. Florida marine shells. Tokyo, Charles E. Tuttle Co., $3^{\text {rd }}$ ed., 170p.

Warmke, G.L. \& R.T. Аввотт. 1962. Caribbean seashells. Pennsylvania, Livingston Publishing Co., $2^{\text {nd }}$ ed., $348 \mathrm{p}$.

WEBB, W.F. 1936. Haudbook for shell collectors, 4. ed. Rochester, 291 p.

WORK, R.C. 1969. Systimatics, ecology and distribuitun of the mollusks of Los Roques, Venezuela. Bull. Mar. Sci. 19 (3): 614-71i.

Recebido em 24.VIII.2001; aceito ein C5.V!.2002. 